\title{
Rheology of Semi-Solid Fresh Cement Pastes and Mortars in Orifice Extrusion
}

\author{
Xiangming Zhou', Zongjin $\mathrm{Li}^{2}$, Mizi Fan ${ }^{3}$, and Huapeng Chen ${ }^{4}$ \\ ${ }^{1}$ School of Engineering and Design, Brunel University, Uxbridge, Middlesex, United \\ Kingdom UB8 3PH Tel: +44 1895266 670, Fax: +44 1895269 782, Email: \\ Xiangming.Zhou@brunel.ac.uk. \\ ${ }^{2}$ Department of Civil and Environmental Engineering, Hong Kong University of Science and \\ Technology, Clear Water Bay, Kowloon, Hong Kong, Tel: +852 2358 8751, Fax: +852 2358 \\ 1534, Email: zongjin@ust.hk. \\ ${ }^{3}$ School of Engineering and Design, Brunel University, Uxbridge, Middlesex, United \\ Kingdom UB8 3PH Tel: +44 1895266 466, Fax: +44 1895269 782, Email: \\ Mizi.Fan@brunel.ac.uk. \\ ${ }^{4}$ School of Engineering, University of Greenwich at Medway, Chatham Maritime, Kent, \\ United Kingdom ME4 4TB Tel: +44 1634883 031, Fax: +44 1634883 153, Email: \\ h.chen@greenwich.ac.uk.
}

Abstract: Short fiber-reinforced semi-solid fresh cement pastes and mortars, tailored for extrusion, have much lower water-to-binder ratio and higher viscosity than normal cement pastes or mortars. The rheology of these pastes or mortars cannot be characterised by traditional rheology test methods suitable for normal fresh cement pastes or mortars with much greater water-to-binder ratio and lower viscosity. In this paper, orifice extrusion is employed to calibrate rheology of the semi-solid fresh cement mortar. An analytical model is developed for orifice extrusion of semi-solid pastes and mortars obeying a rigid-viscoplastic constitutive relationship, von-Mises yield criterion and the associated flow rule. Orifice extrusion results are interpreted using the analytical model and the established experiment data interpretation method and the associated rheological parameters are derived for the semisolid fresh cement mortar. This study provides a simple analytical model, together with experiment and data interpretation methods, for characterizing the complex intrinsic rheological behaviour of semi-solid fresh cement pastes or mortars.

Keywords: Constitutive rheological model; Extrusion; Fresh cement paste or mortar; Flow stress; Rheology; Semi-solid; Strain rate; Viscoplastic 


\section{Introduction}

Extrusion is a common material processing method throughout ceramic, mechanical, chemical and pharmaceutical industries for traditional products such as bricks, tiles, and pipes and for advanced products such as honeycomb panels, etc. The extrusion technology has been successfully introduced into cement and concrete industries, as an economical, efficient and environmental-friendly materials processing method, for manufacturing high-performance fiber-cement building materials and products [1-8]. To cement and concrete industries, the extrusion technique enables the flexibility in fast fabricating building products with complicated shapes, for example, finely structured honeycomb panels, window and door frames, wave-shaped roof tiles etc. without the needs of moulds which could largely reduce production cost. Qian et al. found that short discrete fibers can be aligned along extrusion direction [9], so that the extrusion technique can largely improve mechanical performance of fiber-reinforced cement composites [5,10]. The extrusion technique can produce fiber reinforced cement composites with a well consolidated matrix and good fiber packing, resulting in low porosity and strengthening of the fiber matrix bond [10].

A successful extrusion process of fiber cement products largely depends on the rheological properties of the fiber-reinforced semi-solid fresh cement paste or mortar in extrusion as well as the extrusion hardware system [11]. Though lots of extrusion practices have been successful on fiber-reinforced cement-based materials and products, limited research has been carried out on mechanical/rheological behavior of extrudable fresh cement paste or mortar itself, which is highly concentrated and semi-solid. So far, the rheological behavior of these fiber-reinforced semi-solid fresh cement pastes or mortars has not been well understood due to the complex elastic, plastic and viscous properties combined. It should be noted that the semi-solid fresh cement pastes or mortars suitable for extrusion, investigated in this study, are largely different from traditional fresh cement pastes, mortars, suspensions, 
slurries, or concretes which normally have greater water-to-binder ratio, much lower viscosity and exhibit better fluidity. Short fiber-reinforced fresh cement pastes or mortars for extrusion purpose are dough-like materials normally incorporating rheology enhancing admixture, such as Methocel, to increase their viscosity and cohesion, which exhibit almost no fluidity, but high cohesion and viscoplastic behavior under normal conditions [12-14]. In addition, the discrete short fibers largely increase the viscosity and cohesion and reduce the fluidity of the fresh cement pastes or mortars tailored for extrusion. It should be noted that traditional rheology test methods, suitable for flowable fresh concretes, cement pastes or mortars, may not be appropriate for these highly concentrated, cohesive and semi-solid fresh cement-based materials for extrusion purpose [15].

So far, there are limited quantitative data available for describing rheological behavior of the highly concentrated semi-solid fresh cement pastes or mortars for extrusion purpose, mainly due to the lack of appropriate rheology test methods. Alfani and Guerrini [15] reviewed several most promising 'non-traditional' rheology test methods for concentrated and cohesive extrudable fresh cement-based materials and found that those test methods were initially developed for materials like plastics, rubber, clays, soils and metals, rather than traditional cement pastes or mortars.

Ram extrusion is frequently utilized to characterize rheological properties of semi-solid pastes in ceramic and chemical engineering. Typical configuration of ram extrusion involves measuring the extrusion pressure required to extrude paste in a barrel with larger diameter through a die land with smaller diameter and certain length. This technique was adopted for characterizing rheological behavior of fiber-reinforced extrudable fresh cement pastes and mortars $[12,16]$. In those studies, the extrusion behavior of the highly concentrated fresh cement pastes or mortars was described in terms of the relationship between extrusion pressure and material flow velocity using an empirical phenomenological model proposed by 
Benbow and Bridgewater [17]. It should be noted that these studies only provide apparent rheological properties, not intrinsic constitutive behavior, for fiber-reinforced semi-solid cement pastes or mortars, which will be discussed in details later in this paper.

Capillary extrusion was also utilized for calibrating the rheological behavior of highly viscous semi-solid fresh cement pastes and mortars for extrusion purpose by Alfani et al. [15], Zhou and Li [18], and Kuder and Shah [19]. It was found by Zhou and Li [18] that the postyield steady-state shear flow behavior of the semi-solid extrudable fresh cement pastes and mortars can be described by the Herschel-Bulkley relationship between shear flow stress and shear strain rate while the study conducted by Alfani et al. [15] indicated that the shear flow behavior of the extrudable fresh cement-based materials obeys a power-law relationship which is the simplified Herschel-Bulkley relationship with the shear yield strength equal to zero.

Squeezing flow test was investigated as an extrusion-ability-identifying tool to characterize rheology of highly concentrated firm fresh cement pastes and mortars by Toutou et al. [13]. It was concluded that extrusion ability requires a balance between processing environment and material rheological properties. The plastic behavior of the firm cement pastes and mortars for extrusion process was described by two different models: the perfect plastic model following the von Mises yield criterion and the plastic stress hardening model following the Drucker-Prager criterion, respectively [13]. It should be noted that these investigations do not consider the time- or rate-dependent effect and are only applicable to material processes in which the deformation rate is low, such as upsetting and squeezing flow, and may not be appropriate for high-rate extrusion process. Upsetting test was utilized to derive the quantitative relationship among the plastic flow stress, true strain and true strain rate of the short fiber-reinforced highly concentrated semi-solid fresh cement mortar for extrusion purpose at low strain rate [14]. It was concluded that the strain rate-hardening effect 
dominates the constitutive behavior of the extrudable semi-solid fresh cement pastes [14]. Theoretical solution of squeezing flow was presented by $\mathrm{Li}$ and $\mathrm{Li}$ [20] for semi-solid fresh cement pastes and mortars obeying the Herschel-Bulkley constitutive relationship. They concluded that the constitutive behavior of the highly concentrated fresh cement pastes and mortars for extrusion purpose is mainly governed by rheological (strain rate-dependent) effect while plastic (strain-dependent) effect is not significant, especially when the semi-solid fresh cement pastes and mortars exhibit large deformation and/or strain. Recently, the squeezing flow test was adopted to evaluate the rheological behavior of cement-based mortars with relatively high entrained air but low water contents exhibiting highly viscous under different squeezing rates by Cardoso et al. [21]. All these studies indicate that it is possible to evaluate different aspects of the constitutive behavior of the highly viscous semisolid fresh cement pastes or mortars for extrusion purpose by combining several 'nontraditional' test methods, but nevertheless the best test method is through extrusion itself, among which orifice extrusion is probably the simplest one to conduct.

A computational elasto-viscoplastic constitutive model was successfully established for short fiber-reinforced highly concentrated semi-solid fresh cement pastes and mortars for extrusion purpose [22]. This constitutive model is based on the consistency viscoplasticity [23] and features the von-Mises yield criterion, the associated flow rule and nonlinear strain rate-hardening law with the Herschel-Bulkley relationship between the flow stress and strain rate. The rate-form constitutive model is integrated into an incremental formulation which enables it to be implemented into numerical frameworks, like finite element formulation [11, 24]. For each of the physical phenomena included in the elasto-viscoplastic constitutive model, one or more material parameters are required, which need to be determined from those 'non-traditional' test methods, i.e., capillary extrusion, upsetting, squeezing flow etc., described above plus appropriate experimental data interpretation techniques. However, the 
data interpretation technique could be very complicated. For instance, the data interpretation processes for upsetting tests [14], capillary extrusion [18], and squeezing flow [20] all require a large amount of experiment data involving different geometries and the data interpretation processes themselves are very complex and time-consuming. Besides, it may need several 'non-traditional' test methods to be combined together in order to obtain various aspects of the constitutive behavior of the semi-solid fresh cement pastes or mortars [22], making their applications very limited. A simple material test method with relatively simple data interpretation procedure is thus greatly needed to obtain various aspects of the complex constitutive behavior of the fiber-reinforced highly concentrated semi-solid fresh cement pastes or mortars for extrusion purpose.

In this study, orifice extrusion, with the geometry based on a cylindrical square-ended barrel with orifices of various diameters but zero-length, is adopted to characterize rheological properties of short fiber-reinforced highly concentrated fresh cement mortars for extrusion purpose. Experimental results are interpreted by an analytical model developed for orifice extrusion of semi-solid materials obeying a rigid-viscoplastic constitutive relationship in the format of Herschel-Bulkley equation incorporating incompressibility, the von-Mises yield criterion and the associated post-yield flow rule. The associated constitutive material parameters are derived for the highly concentrated semi-solid fresh cement mortar for extrusion purpose. Compared with capillary extrusion, upsetting or squeezing flow analyses, the present orifice extrusion analysis is relatively simple and requires much less experimental data and the data interpretation process is much less time-consuming. It is a promising technique for characterizing the complex constitutive behavior of short fiber-reinforced highly concentrated semi-solid fresh cement pastes and mortars, which consequently helps to tailor their rheology for successful extrusion processes. Furthermore, by using the analytical 
model for orifice extrusion, it is possible to employ only one extrusion geometry to derive the constitutive rheological behavior of the semi-solid fresh cement pastes or mortars.

\section{Theoretical Approach}

\subsection{Constitutive rheological models for semi-solid fresh cement pastes and mortars}

Previous studies have indicated that short fiber-reinforced highly concentrated fresh cement pastes and mortars for extrusion purpose exhibit complex elastic, viscous and plastic constitutive behaviour $[12,14,15,20,22]$. In theory, the rheological behaviour of short fiberreinforced highly concentrated semi-solid cement pastes and mortars, with the addition of rheology enhancing admixture, should be described as elasto-viscoplastic [22]. However, for most materials in extrusion, plastic strain is much greater compared with elastic strain. Under these conditions, these materials may be treated as rigid-viscoplastic.

In ram and capillary extrusion, due to no wall available for developing the slip layer in the die-entrance (orifice) region, the semi-solid material is forced to shear and deform plastically without a thin layer of slip flow between the bulk material flow and the extruder wall [27, 28], therefore forming a combined differential (shear) flow plus an extensional (plastic) flow, i.e., viscoplastic flow. If the relationship among flow stress, strain and strain rate of the material flow in the die land-entrance (orifice) region can be obtained, it could be used to describe the constitutive rheological properties of the semi-solid paste or mortar. From this point of view, ram extrusion through dies with zero-length, i.e. orifice extrusion, is a useful technique for characterizing intrinsic material behaviour of highly concentrated semi-solid cohesive fresh cement pastes or mortars for extrusion purpose. A schematic diagram of orifice extrusion with square-ended geometries is shown in Fig. 1, which also indicates the material flow lines from the upstream towards the downstream in an orifice extruder. For square-ended geometries, static zones, in which the material flow velocity is 
zero, exist at the flow exit region and the flow lines converge towards the orifice as indicated in Fig. 1. The static zones are bounded by slip planes, which form approximately a conical exit geometry at certain angle between $0^{0}$ and $90^{\circ}$ to the axis of symmetry of the orifice extruder.

It has been found in previous studies [22] that the constitutive rheological behaviour of the semi-solid cohesive fresh cement pastes or mortars for extrusion purpose can be described by a generalized uniaxial-form Herschel and Bulkley relationship as:

$$
\sigma=\sigma_{0}+k\left(\varepsilon^{v p}\right)^{n} \quad \text { for } \sigma \geq \sigma_{0}
$$

where $\sigma, \sigma_{0}, k$ and $n$ are the equivalent uniaxial flow stress, the uniaxial yield flow stress, the uniaxial flow consistency and the uniaxial flow index, respectively, and $\varepsilon^{v p}$ is the equivalent viscoplastic strain rate. It can be seen from the constitutive rheological model Eq. (1), for materials design purpose, the effects of changes in individual ingredients on overall rheology of the extrudable semi-solid fresh cement pastes or mortars can be reflected by the changes in the three independent intrinsic material constants $\sigma_{0}, k$ and $n$. A stiffer semisolid fresh cement paste or mortar suitable for extrusion normally has a greater yield flow stress $\sigma_{0}$ which has to be overcome in order to initiate material flow in extrusion. A stiffer fresh cement paste or mortar extrudate normally has a lower water-to-binder ratio, higher solid contents, greater rheology enhancing admixture dosage, and/or greater cohesion. For materials design purpose, the yield flow stress $\sigma_{0}$ can be measured using shear box test [15] or upsetting test [14]. On the other hand, a semi-solid fresh cement paste or mortar suitable for extrusion with greater flow resistance, once the material flow has been initiated, usually has greater flow consistency $k$ and flow index $n$. These two intrinsic material parameters mainly determine the steady state flow behavior of the semi-solid fresh cement pastes or mortars in continuous extrusion process. Semi-solid fresh cement pastes and mortars with 
greater water-to-binder ratio and/or greater fiber volume ratio usually possess a greater flow consistency $k$ and/or flow index $n$. For materials design purpose, these two parameters can be obtained through capillary extrusion test [18] or squeeze flow test [13, 20]. It should be noted that, as reviewed in Section 1 Introduction, it needs a series of capillary extrusion tests [18], upsetting tests [14] and squeeze flow tests $[13,20]$ involving with various geometries and/or processing rates in order to quantitatively determine the material constants $\sigma_{0}, k$ and/or $n$. Besides, the experimental data interpretation methods, for capillary extrusion test [18], upsetting test [14] and squeeze flow test $[13,20]$, respectively, could be very complicated. On the other hand, in this study a relatively simple experiment, orifice extrusion, is presented with the associated data interpretation method to quantify the three material constants $\sigma_{0}, k$ and $n$ together but much less experimental data are required for this purpose. The data interpretation method for orifice extrusion is much simpler which can benefit materials design by largely reducing try-and-error efforts on tailoring individual ingredients in semisolid fresh cement pastes or mortars to enable a successful extrusion process.

\subsection{Analytical models for orifice extrusion of semi-solid pastes and mortars}

The theoretical analyses of orifice extrusion are mainly provided by classical plasticity theory with the additional consideration of rate-dependent effect of material flow in high deformation rates. The most widely used method for analyzing orifice extrusion data of semi-solid paste-like materials is based on an equation proposed by Benbow et al. [30], Benbow and Bridgwater [17] as

$$
P=2\left(\sigma_{0}^{\prime}+\alpha V^{j}\right) \ln \left(\frac{D_{0}}{D}\right)
$$


where $P$ is the orifice extrusion pressure, $\sigma_{0}^{\prime}$ is the initial orifice 'yield stress' when the paste flow velocity approaches zero, $V$ is the mean paste flow velocity in the orifice, $D_{0}$ and $D$ are the barrel diameter and the orifice diameter (see Fig. 1), respectively, and $\alpha$ and $j$ are two fitting parameters. This is an empirical extension of the ideal work equation used in engineering plasticity for rate-independent rigid-plastic materials. Though this equation provides reasonably satisfactory predictions for orifice extrusion data of many semi-solid paste-like materials, it is formulated in terms of flow velocity, rather than the strain and/or strain rate of the paste-like material in extrusion. As a result, it contains the velocity coefficient, $\alpha$, in Eq. (2), which is not an intrinsic material parameter. Besides, the initial orifice 'yield stress', $\sigma_{0}^{\prime}$, is a parameter obtained from fitting exercise, i.e., by interpreting orifice extrusion pressure at the paste flow velocity equal to zero, which is impossible to achieve in real experiment. It is not an intrinsic material parameter that is physically meaningful. This relationship is basically a physically phenomenal model for orifice extrusion and it is actually irrespective of material constitutive properties. Thus its accuracy is questionable. For instance, Zheng et al [25] found that the orifice extrusion pressure also depends on diameter of orifice which is consistent with the conclusions drawn by Horrobin and Nedderman [31] from numerical analysis. Since Benbow-Bridgwater model, i.e. Eq. (2), does not contain intrinsic material parameters, the interpretation of orifice extrusion data using this model does not enable any intrinsic material parameter of the highly concentrated semi-solid paste-like material to be derived. A more advanced and fundamental analytical model for orifice extrusion of semi-solid paste-like material is thus required.

Using the spherical coordinates shown in Fig. 2, Gibson [32] developed an analytical equation 


$$
P=k_{u}\left(\frac{2 V}{D}\right)^{n}\left[\frac{2}{3 n}\left(\sin \theta_{\max }\left(1+\cos \theta_{\max }\right)\right)^{n} \times\left(1-\left(\frac{D}{D_{0}}\right)^{3 n}\right)+\Phi\left(n, \theta_{\max }\right)\right]
$$

for predicting the orifice extrusion pressure of semi-solid paste-like material obeying the power-law constitutive model based on a spherically convergent flow, where $\theta_{\max }$ is the angle of the cone formed by the slip planes (also see Fig. 2). The term $\Phi\left(n, \theta_{\max }\right)$ is an end effect to take into account the transition from convergent to non-convergent flow and kinematic effect of the semi-solid paste-like material out of orifice, i.e., the material flow exiting the die entrance still carries some kinematic energy which is not able to be taken into account when deriving Eq. (3).

Using the same spherical coordinate system adopted by Gibson [32], Basterfield et al. [28] proposed a framework for developing an analytical model for orifice extrusion of Herschel-Bulkley type paste-like materials. In this theoretical framework, the die entrance flow field is assumed to be radially convergent in the region $0<\theta<\theta_{\max }, r_{\min }<r<r_{\max }$, where $\theta_{\max }, r_{\min }$, and $r_{\max }$ depend on the orifice and barrel diameters (see Fig. 2). The theoretical analysis is based on the following assumptions: (1) incompressible material flow; (2) the material flow is sufficiently slow that inertial terms may be neglected; and (3) irrotational so that the rectangular material element shown in Fig. 2 stretches but does not rotate. Since both the sections of the barrel and the orifice perpendicular to the flow direction are circular and axis-symmetric, it is reasonable to assume that the velocity components, $u_{(\phi)}$ and $u_{(\theta)}$, in the angular directions $\phi$ and $\theta$, respectively, are equal to zero so that only the velocity component in the radial direction, $u_{(r)}$, is not equal to zero, which varies with respect to the radial position, $r$, only. Based on the assumption of incompressibility for the semi-solid paste-like material, the volumetric flow rates passing the spherical cap and the orifice can be equated: 


$$
2 \pi r\left(r-r \cos \theta_{\max }\right) u_{(r)}=\pi\left(r_{\min } \sin \theta_{\max }\right)^{2} V
$$

where $V$ is the material flow velocity along the axis of symmetry passing the orifice. Equation (4) yields the radial velocity of the material flow in the barrel as

$$
u_{(r)}=\frac{r_{\min }^{2} \sin ^{2} \theta_{\text {max }} V}{2 r^{2}\left(1-\cos \theta_{\max }\right)}
$$

So that, in a spherical coordinate system, the strain rate tensor is given by

$$
\dot{\varepsilon}=\left[\begin{array}{lll}
\frac{\partial u_{(r)}}{\partial r} & & \\
& \frac{u_{(r)}}{r} & \\
& & \frac{u_{(r)}}{r}
\end{array}\right]=-\frac{r_{\min }^{2} \sin ^{2} \theta_{\max } V}{r^{3}\left(1-\cos \theta_{\max }\right)}\left[\begin{array}{ccc}
2 & & \\
& -1 & \\
& & -1
\end{array}\right]
$$

Based on the von Mises yield criterion, the equivalent viscoplastic strain rate, $\varepsilon^{v p}$, can be calculated by

$$
\stackrel{-}{\varepsilon^{v p}}=\sqrt{\frac{2}{3} \dot{\varepsilon}^{v p}: \dot{\varepsilon}^{v p}}
$$

while the von-Mises equivalent flow stress, $\bar{\sigma}$, is given by

$$
\bar{\sigma}=\sqrt{\frac{3}{2} S: S}
$$

where $S=\sigma-\frac{1}{3} \operatorname{tr}(\sigma)$ is the deviatoric stress. When the paste-like material evolves according to the associated flow rule after yielding, the following relationship exists: 


$$
\frac{S}{\bar{\sigma}}=\frac{\dot{\varepsilon}}{\varepsilon^{v p}}
$$

On the other hand, the constitutive relationship, Eq. (1), can be re-written in a more general formulation as:

$$
\bar{\sigma}=\overline{\sigma_{0}}+k\left(\dot{\varepsilon}^{v p}\right)^{n} \quad \text { for } \bar{\sigma} \geq \overline{\sigma_{0}}=\sqrt{3} \tau_{0}
$$

The equilibrium equation for the rectangular material element (as shown in Fig. 2) in a spherical coordinate is given by

$$
\begin{aligned}
& \frac{\partial}{\partial r} \sigma_{r r}+\frac{2}{r} \sigma_{r r}-\frac{1}{r}\left(\sigma_{\phi \phi}+\sigma_{\theta \theta}\right)=0 \\
& \sigma_{\theta \theta}-\sigma_{\phi \phi}=0
\end{aligned}
$$

From Eqs. (6), (7) and (9), the solution of Eq. (11) is seen as

$$
\begin{aligned}
& \sigma_{r r}=-p+\frac{2}{\sqrt{3}} \bar{\sigma} \\
& \sigma_{\phi \phi}=\sigma_{\theta \theta}=-p-\frac{1}{\sqrt{3}} \bar{\sigma}
\end{aligned}
$$

in which $p$ is the hydrostatic stress in the material flow in orifice extrusion.

The extrusion load needed at upstream in orifice extrusion to drive the material flow is the total transmitted load, $L$, divided by the area such that:

$$
L=-\int_{\theta=0}^{\theta_{\max }} 2 \pi r_{\max }^{2} \sin \theta \sigma_{r r}\left(r_{\max }\right) \cos \theta d \theta=-\pi r_{\max }^{2} \sigma_{r r}\left(r_{\max }\right) \sin ^{2} \theta_{\max }
$$

which enables the extrusion pressure at upstream to be determined as 


$$
P=\frac{4 L}{\pi D_{0}^{2}}=\frac{L}{\pi r_{\max }^{2} \sin ^{2} \theta_{\max }}=-\sigma_{r r}\left(r_{\max }\right)
$$

By introducing the generalized Herschel-Bulkley constitutive law, i.e., Eq. (10), of the pastelike material and neglecting the end effect, $\Phi\left(n, \theta_{\max }\right)$ in Eq. (3), an analytical relationship, between the orifice extrusion pressure, $P$, and the mean material flow velocity, $V$, through the orifice along the axis of symmetry, is given by [28]:

$$
P=-\sigma_{r r}\left(r_{\max }\right)=2 \sigma_{0} \ln \frac{D_{0}}{D}+A k\left(\frac{V}{D}\right)^{n}\left(1-\left(\frac{D}{D_{0}}\right)^{3 n}\right)
$$

where

$$
A=\frac{2}{3 n}\left(\sin \theta_{\max }\left(1+\cos \theta_{\max }\right)\right)^{n}
$$

It should be noted that, different from the model proposed by Benbow et al. [30], the new analytical model, Eq. (15), for orifice extrusion is based on the intrinsic material parameters, $\sigma_{0}, k$ and $n$, of the semi-solid paste-like material itself. Thus by interpreting orifice extrusion data with various barrel and/or orifice diameters and material flow velocities with this equation, the constitutive material parameters of the semi-solid paste-like material can be derived. Certainly, in Eq. (16), the maximum convergent flow angle, $\theta_{\max }$, is still unknown. By speculating that the influence of the dead zones is such as to produce a slip plane of zero shear stress, which is roughly conical, Basterfield et al. [28] found that the angle $\theta_{\max }$ is in the range $40^{\circ}-60^{\circ}$ for most paste-like material flow, which is consistent with the detailed finite element analysis presented by Horrobin and Nedderman [31]. Furthermore, it was found that the sensitivity of the parameter, $A$, in Eqs. (15) and (16) to the choice of $\theta_{\max }$ is very small for typical values of $n$ for semi-solid cohesive paste-like materials. Therefore, in this study, a value of $45^{\circ}$ was adopted for $\theta_{\max }$ when comparing experimental data with 
analytical predictions using Eq. (15) for the short fiber-reinforced highly concentrated semisolid fresh cement mortar for extrusion purpose.

With the orifice extrusion analytical model Eq. (15), it will benefit materials design of extrudable fresh fiber cement pastes or mortars and quantify the role of individual ingredients on rheology of extrudate. Given two fiber cement pastes or mortars subjected to the same orifice extrusion test, i.e., with the same $\mathrm{D}_{0}, \mathrm{D}$, and $\mathrm{V}$, the paste or mortar requiring greater orifice extrusion pressure may demonstrate lower extrudability and may generally have greater $\sigma_{0}, k$ and/or $n$. In addition, the role of individual ingredients is implicitly reflected by Eq. (15). In order to quantity the role of individual ingredients, orifice extrusion test with only one die geometry under one extrusion velocity is needed. Once the experimental data is interpreted by Eq. (15), the effects of individual ingredients on the rheology of extrudable fiber cement paste or mortar can be quantified explicitly by the three material parameters $\sigma_{0}$, $k$ and $n$. For instance, a longer fiber length and a greater fiber volume ratio will result in higher flow consistency $k$ and flow index $n$.

\section{Experiment, Results and Discussion}

\subsection{Experiment}

Orifice extrusion was conducted on the short fiber-reinforced semi-solid fresh cement mortar, with the mix formulation shown in Table 1 . The basic constitutive materials for making the cement mortar included Type I OPC (Ordinary Portland Cement) and slag with the weight ratio of $1: 1$ as the binder, 6mm-long PVA (Polyvinyl Alcohol) fiber with an average diameter of $14 \mu \mathrm{m}$, two types of silica sands (denoted as SS1 and SS2 with the nominal diameters of 300-600 $\mu \mathrm{m}$ and 90-150 $\mu \mathrm{m}$, respectively) from David Ball Comp. Ltd with a weight ratio of 8:5 as aggregates, Methocel powder produced by Dow Chemical Comp. 
Ltd as the rheology enhancing admixture and ADVA solution supplied by W. R. Grace Ltd as the superplasticiser. The chemical compositions of the OPC and slag are shown in Table 2. Slag was added to replace part of the OPC for the purpose to increase setting time, thus workablility, of the semi-solid fresh cement mortar. The water-to-binder weight ratio was 0.25 while that of the silica sand-to-binder was 0.325 . The dosage of ADVA solid powder in the mixture was $0.25 \%$ in weight of the binder. The ADVA solid power was incorporated into the mixture in the form of an aqueous solution with a concentration of $30 \%$ by weight as supplied by the manufacturer. The PVA fibers were provided by Kuraray Co. Ltd. and their physical and mechanical properties are shown in Table 3. The amount of PVA fibers incorporated was $2 \%$ by volume of the readily mixed fresh cement mortar.

The procedure for preparing the fresh cement mortar for extrusion is as follows. First, the binder powders (cement and slag), the fibers and the Methocel powder were mixed for 3 minutes in dry state at the lowest gauge of a stand-alone Hobart planetary mixer. Then, water (with superplasticiser) was added into the mixture and mixed with other components for another 3 minutes. Once the dry powders were sufficiently moistened, the fresh composite was subjected to high shear mixing under a higher speed till a dough-like semi-solid fresh cement mortar was produced, which was then lumped into the cylindrical barrel, with the inner diameter of $80 \mathrm{~mm}$ and the height of $150 \mathrm{~mm}$, of an in-house orifice extruder (as shown in Fig. 3) and was ready for extrusion.

A typical orifice extrusion proceeded as follows. The barrel was filled with a certain amount in weight of ready-mixed fresh cement mortar up to its brim. One end of the piston 'A' (see Fig. 3) was connected to a MTS materials test system while the other end was positioned in the barrel with the lower surface of the piston in direct contact with the mortar. The barrel was placed on Plate ' $B$ ' which was fastened on four vertical steel rods. A plate 'C' was fixed at the bottom of four steel rods and connected to the actuator of the MTS materials 
test system. When the actuator of the MTS system was moving up to press the mortar inside the barrel, the piston 'A' prevented the up movement of the cement mortar. Subsequently, the mortar was extruded out of the barrel from the circular orifice underneath. At the beginning of each test, the mortar was driven with a velocity of $0.1 \mathrm{~mm} / \mathrm{s}$ to a displacement of $20 \mathrm{~mm}$, which was found to be long enough to achieve steady state with defect-free extrudate. Then the extrusion process continued under a designated constant driving velocity. Each extrusion process was repeated three times under the same driving velocity and the average extrusion load at the steady state was taken as the representative extrusion load. Fig. 4 shows an orifice extrusion of the highly concentrated semi-solid fresh cement mortar in process.

In this study, the semi-solid fresh cement mortar in the cylindrical barrel of the orifice extruder was driven by an MTS materials test system at a series of velocities, respectively, listed in Table 4 through three different orifices with the diameter of 8,12 and $15 \mathrm{~mm}$, respectively. All the orifices were effectively sharp-edged dies with the lengths equal to zero. The corresponding mean material flow velocities along the axis of symmetry of the cylindrical extruder through orifice are also listed in Table 4. It should be noted that velocity of the mortar flow at orifice was not directly measured in experiment. Rather it was calculated based on the assumption that the semi-solid highly viscous fresh cement pastes and mortars for extrusion purpose are incompressible which is an assumption widely taken for highly viscous materials.

\subsection{Results and discussion}

An example of orifice extrusion pressure with respect to time is shown in Fig. 5, which indicates that the orifice extrusion pressure increased at the beginning of the test till the semisolid fresh cement mortar started to be extruded out of the orifice. Then the extrusion 
pressure remained almost constant, i.e., reaching steady state, under constant driving velocity. After certain period, the driving velocity was reduced to zero and the extrusion process was stopped. Then the fresh cement mortar in the barrel began to relax due to the nature of its viscous and plastic behavior. The pressure during relaxation gradually leveled off and this value was taken as the relaxation pressure. It should be noted that no pressure sensor was used in the system. The extrusion pressure shown in Fig. 5 was actually the driving pressure applied by the piston at the upstream of the orifice extrusion flow. The steady-state orifice extrusion pressures are plotted as a function of the value, V/D, in Figs. 6-8 for each orifice diameter, respectively. It should be noted that the value of V/D designates the mean shear rate of the cement mortar when passing the orifice, which is somehow irrespective of diameter of orifice, while, on the other hand, the mean material flow velocity, $\mathrm{V}$, does depend on diameter of orifice. So it is more meaningful to plot the orifice extrusion pressure as a function of V/D rather than $\mathrm{V}$ when comparing orifice extrusion data obtained from different orifice diameters. It can be seen from Figs. 6-8 that the pressures for greater mean shear rate are greater than those for smaller mean shear rate, suggesting that the highly concentrated semi-solid fresh cement mortar exhibits strain rate-hardening behavior.

The analytical model, Eq. (15), was fitted to the orifice extrusion data using a nonlinear least squares regression analysis in Originlab to determine the values of the associated three independent material parameters, i.e., $\sigma_{0}, k$ and $n$, so that the root mean square (RMS) error is minimized. The fitted curves, in the formulation of Eq. (15), are also shown in Figs. 6 to 8, respectively, for each orifice diameter. The constitutive material parameters, $\sigma_{0}, k$ and $n$, from the fitting exercise with the analytical model, Eq. (15), are shown in Table 5 for each orifice diameter for the highly concentrated semi-solid fresh cement mortar for extrusion with the mix proportion shown in Table 1. It can be seen from Table 5 that the values of the RMS error is smaller for tests with greater orifice diameter. The fitting exercise yields greater 
uniaxial yield flow stress, $\sigma_{0}$, but smaller uniaxial flow consistency, $k$, for smaller orifice diameter. It can be found that the values of $\sigma_{0}$ and $k$ obtained from fitting exercise do not differ much from the orifice extrusion results with different orifice diameters. On the other hand, the uniaxial flow index ranges between 0.36 and 0.44 obtained for the three orifice diameters and the value obtained from each orifice diameter is very close to each other. By considering that the rheological properties of the semi-solid fresh cement mortar may change as the progression of cement hydration during preparation and execution of extrusion, resulting in that the constitutive material parameters of the fresh cement mortar may change accordingly with respect to time and the progression of cement hydration, which was not able to be taken into account in the analytical model described in this study, the results shown in Table 5 obtained from fitting exercise from different orifice diameters are acceptable and consistent. Thus, in practice, it is possible to use only one orifice diameter in a series of orifice extrusion tests involving in different extrusion velocity to derive the relevant constitutive material parameters, i.e., $\sigma_{0}, k$ and $n$, of highly viscous semi-solid fresh cement pastes and mortars for extrusion purpose to reduce experimental work needed for characterizing rheology of those pastes or mortars.

\section{Conclusions}

(1) Short discrete fiber-reinforced fresh cement pastes and mortars tailored for extrusion exhibit largely different rheological behavior from traditional fresh cement pastes, mortars or concretes, which normally possess much greater water-to-binder ratio, lower viscosity and better fluidity. The traditional rheology test methods suitable for flowable fresh cement pastes, mortars and concretes may not be appropriate to the highly viscous semi-solid fresh cement pastes or mortars for extrusion purpose. 
(2) In this study, orifice extrusion is employed as a non-traditional rheology test method to characterize the complex rheological properties of semi-solid fresh cement mortars with mix formulation suitable for extrusion. An analytical model is successfully developed for orifice extrusion of semi-solid paste-like materials obeying the Herschel-Bulkley viscoplastic constitutive law, the von-Mises yield criterion and the associated flow rule. Different from those phenomenological models for orifice extrusion, which are established normally irrespective of the intrinsic properties of the material in extrusion, this analytical model includes only physically meaningful material parameters. It can be used for deriving the constitutive material parameters of the semi-solid pastes or mortars for extrusion.

(3) A series of orifice extrusion tests are conducted on the highly concentrated semi-solid fresh cement mortar under various extrusion velocities through orifices with different diameters. The analytical model is then utilized to interpret the experimental data. The associated rheological parameters are derived for the semi-solid fresh cement mortar. It can be concluded that the analytical model provides an effective data interpretation approach for orifice extrusion in quantifying the intrinsic rheological behaviour of extrudable cement pastes or mortars. More importantly, by using the analytical model for orifice extrusion, it is possible to employ only one extrusion geometry to derive the constitutive rheological behavior of the semi-solid fresh cement pastes or mortars. Compared with other methods, it will largely reduce experimental efforts required for quantifying rheology of semi-solid fresh fiber cement pastes or mortars suitable for extrusion.

(4) The orifice extrusion itself is a simple but promising rheology test method for characterizing the complex rheological behaviour of highly concentrated semi-solid fresh cement pastes or mortars. By combining with the analytical model, orifice extrusion can be used for tailoring mix proportions of semi-solid fresh cement pastes or mortars to achieve successful extrusion processes. 


\section{ACKNOWLEDGEMENT}

The financial support from the Brunel Research \& Innovative Enterprise Fund under the award LBK909(904/2009), from the European Commission $7^{\text {th }}$ Framework Programme through the call FP7-NMP-2010-SMALL-4 under the grant 262954, and from the China Ministry of Science and Technology under grant 2009CB623200 is gratefully acknowledged.

\section{REFERENCE}

1. Shao Y, Marikunte S, Shah SP. Extruded fiber-reinforced composites. Concr Int 1995;17(4):48-52.

2. Shao Y, Shah SP. Mechanical properties of PVA fiber reinforced cement composites fabricated by extrusion processing. ACI Mater J 1997;94(6):555-564.

3. Aldea C, Marikunte S, Shah SP. Extruded fiber reinforced cement pressure pipes. Adv Cem Based Mater 1998;8(2):47-55.

4. Li ZJ, Mu B, Chui SNC. Systematic study of properties of extrudates with incorporated metakaolin or silica fume. ACI Mater J 1999;96(5):574-579.

5. Li ZJ, Mu B, Chui SNC. Static and dynamic behaviour of extruded sheets with short fibers. J Mater Civ Engrg ASCE 2001;13(4):248-254.

6. Li ZJ, Zhou XM, Shen B. Fiber-cement extrudates with perlite subjected to high temperatures. J Mater Civ Engrg ASCE 2004;16(3):221-229.

7. Li ZJ, Zhang YS, Zhou XM. Short fiber-reinforced geopolymer composites manufactured by extrusion. J Mater Civ Engrg ASCE 2005;17(6):624-631.

8. Kuder KG, Shah SP. Tailoring extruded HPFRCC to Be Nailable. ACI Mater J 2007;104(5):526-534. 
9. Qian XQ, Zhou XM, Mu B, Li ZJ. Fiber alignment and property direction dependency of FRC extrudate. Cem Concr Res 2003;33(10):1575-1581.

10. Peled A, Shah SP. Processing effects in cementitious composites: extrusion and casting. J Mater Civ Engrg ASCE 2003;15(2):192-199.

11. Zhou XM, Li ZJ. Numerical simulation of ram extrusion process of short fiber-reinforced fresh cementitious composite. J Mech Mater Struct 2009;4(10):1755-1769.

12. Srinivasan R, DeFord D, Shah SP. The use of extrusion rheometry in the development of extruded fiber-reinforced cement composites. Concr Sci Engrg 1999;1(1):26-36.

13. Toutou Z, Roussel N, Lanos C. The squeeze test: a tool to identify firm cement-based material's rheological behaviour and evaluate their extrusion ability. Cem Concr Res 2005;35(10):1891-1899.

14. Zhou XM, Li ZJ. Upsetting tests of fresh cementitious composites for extrusion. J Engrg Mech ASCE 2006;132(2):149-157.

15. Alfani R, Guerrini GL. Rheology test methods for the characterization of extrudable cement-based materials - A review. Mater Struct RILEM 2005;38(2):239-247.

16. Zhou X, Li Z. Characterization of rheology of fresh fiber reinforced cementitious composites through ram extrusion. Mater Struct RILEM 2005;38(1):17-24.

17. Benbow JJ, Bridgwater J. Paste flow and extrusion. Oxford: Clarendon Press, 1993.

18. Zhou XM, Li ZJ. Characterizing rheology of fresh short fiber reinforced cementitious composite through capillary extrusion. J Mater Civ Engrg ASCE 2005;17(1):28-35.

19. Kuder KG, Shah SP. Rheology of extruded cement-based materials. ACI Mater J 2007;104(3):283-290.

20. Li ZJ, Li XY. Squeeze flow of viscoplastic cement-based extrudate. J Engrg Mech ASCE 2007;133(9):1003-1008. 
21. Cardoso FB, John VM, Pileggi RG. Rheological behavior of mortars under different squeezing rates. Cem Concr Res 2009;39(9):748-753.

22. Zhou XM, Li ZJ. A constitutive model for fiber-reinforced extrudable fresh cementitious paste. Comput Concr: An Inter J 2011;8(4):371-388.

23. Ponthot JP. Unified stress update algorithms for the numerical simulation of large deformation elasto-plastic and elasto-viscoplastic processes. Int J Plasticity 2002;18(1):91126.

24. Zhou XM, Li ZJ. Numerical simulations of upsetting process of the fresh fiber-cement paste. J Engrg Mech ASCE 2007;133(11):1192-1199.

25. Zheng J, Carlson WB, Reed JS. Flow mechanics on extrusion through a square-entry die. J Am Ceram Soc 1992;75(11):3011-3016.

26. Khan AU, Briscoe BJ, Luckham PF. Evaluation of slip on capillary extrusion of ceramic pastes. J Eur Ceram Soc 2001;21(4):483-491.

27. Adams MJ, Briscoe BJ, Kamjab M. The deformation and flow of highly concentrated dispersions. Adv Colloid Interf Sci 1993;44:143-182.

28. Basterfield RA, Lawrence CJ, Adams MJ. On the interpretation of orifice extrusion data for viscoplastic materials. Chem Engrg Sci 2005;60(10):2599-2607.

29. Aydin I, Biglari FR, Briscoe BJ, Lawrence CJ, Adams MJ. Physical and numerical modelling of ram extrusion of paste materials: conical die entry case. Comput Mater Sci 2000;18(2):141-155.

30. Benbow JJ, Oxley EW, Bridgwater J. The extrusion mechanics of pastes - the influence of paste formulation on extrusion parameters. Chem Engrg Sci 1987;42(9):2151-2162.

31. Horrobin DJ, Nedderman RM. Die entry pressure drops in paste extrusion. Chem Engrg Sci 1998;53(18):3215-3225. 
32. Gibson AG. Converging dies. In: Collyer AA, Clegg DW, editors. Rheological measurement. Barking: Elsevier, 1988. p. 49-92. 


\section{APPENDIX}

\section{TABLES}

Table 1 Mix formulation of the short fiber-reinforced fresh cement mortar for extrusion

\begin{tabular}{|c|c|c|c|c|c|c|c|}
\hline Cement & Slag & SS1 & SS2 & PVA & Methocel & ADVA & W/B \\
\hline 0.5 & 0.5 & 0.2 & 0.125 & $2 \%$ & $1 \%$ & $0.25 \%$ & 0.25 \\
\hline
\end{tabular}

Note: SS1 and SS2: silica sand with 300-600 $\mu \mathrm{m}$ and 90-150 $\mu \mathrm{m}$ in diameter, respectively, from David Ball Comp. Ltd;

PVA: Polyvinyl alcohol fiber from Kuraray Co. Ltd.; B: binder (Type I ordinary Portland cement + slag); W: water;

ADVA: superplasticiser produced by W.R. Grace (HK) Ltd;

Methocel: rheology enhancing admixture produced by Dow Chemical, USA;

SS1, SS2, Methocel and ADVA are presented in weight ratio of the binder;

PVA fiber is presented in the volume ratio of the readily mixed fresh cement mortar. 
Table 2 Chemical compositions of OPC and slag (\% in weight)

\begin{tabular}{|c|c|c|c|c|c|c|c|c|c|c|}
\hline Binder & $\mathrm{CaO}$ & $\mathrm{SiO}_{2}$ & $\mathrm{Al}_{2} \mathrm{O}_{3}$ & $\mathrm{Fe}_{2} \mathrm{O}_{3}$ & $\mathrm{TiO}_{2}$ & $\mathrm{~K}_{2} \mathrm{O}$ & $\mathrm{Na}_{2} \mathrm{O}$ & $\mathrm{MgO}$ & $\mathrm{LOI}$ & $\mathrm{SO}_{3}$ \\
\hline OPC & 63.12 & 20.83 & 6.28 & 2.47 & 0.21 & 0.61 & 0.25 & 1.16 & - & 2.04 \\
\hline Slag & 39.50 & 28.48 & 12.56 & 1.56 & 0.44 & 0.44 & 0.20 & 7.40 & 0.50 & 8.48 \\
\hline
\end{tabular}


Table 3 Properties of short polyvinyl alcohol (PVA) fibers

\begin{tabular}{|c|c|c|c|c|c|}
\hline $\begin{array}{c}\text { Density } \\
\left(\mathrm{g} / \mathrm{cm}^{3}\right)\end{array}$ & $\begin{array}{c}\text { Tensile strength } \\
(\mathrm{Mpa})\end{array}$ & $\begin{array}{c}\text { Elastic modulus } \\
(\mathrm{Gpa})\end{array}$ & $\begin{array}{c}\text { Length } \\
(\mathrm{mm})\end{array}$ & $\begin{array}{c}\text { Diameter } \\
(\mu \mathrm{m})\end{array}$ & Aspect ratio \\
\hline 1.30 & 1,500 & 36 & 6 & 14 & 430 \\
\hline
\end{tabular}


Table 4 Piston driving velocity and material flow velocity in orifice

\begin{tabular}{|c|c|c|c|}
\hline $\begin{array}{c}\text { Piston driving } \\
\text { velocity (mm/s) }\end{array}$ & \multicolumn{3}{|c|}{ Paste flow velocity at orifice (mm/s) } \\
\cline { 2 - 4 } & $\mathrm{D}=8 \mathrm{~mm}$ & $\begin{array}{c}\mathrm{D}=12 \\
\mathrm{~mm}\end{array}$ & $\mathrm{D}=15 \mathrm{~mm}$ \\
\hline 0.02 & 2 & 0.89 & 0.57 \\
\hline 0.05 & 5 & 2.22 & 1.42 \\
\hline 0.10 & 10 & 4.44 & 2.84 \\
\hline 0.20 & 20 & 8.89 & 5.69 \\
\hline 0.30 & 30 & 13.33 & 8.53 \\
\hline 0.40 & 40 & 17.78 & 11.38 \\
\hline 0.50 & 50 & 22.22 & 14.22 \\
\hline 0.60 & 60 & 26.67 & 17.07 \\
\hline 0.70 & 70 & 31.11 & 19.91 \\
\hline 0.80 & 80 & 35.56 & 22.76 \\
\hline 0.90 & 90 & 40.00 & 25.60 \\
\hline 1.00 & 100 & 44.44 & 28.44 \\
\hline 1.20 & 120 & 53.33 & 34.13 \\
\hline 1.50 & 150 & 66.67 & 42.67 \\
\hline
\end{tabular}


Table 5 Material parameters obtained from fitting experimental data with the analytical model (Eq. (15))

\begin{tabular}{|c|c|c|c|}
\hline $\mathrm{D}(\mathrm{mm})$ & 8 & 12 & 15 \\
\hline $\mathrm{D} / \mathrm{D}_{0}$ & 0.1 & 0.15 & 0.1875 \\
\hline$\sigma_{0}(\mathrm{kPa})$ & $20.55 \pm 2.68$ & $13.73 \pm 1.65$ & $11.29 \pm 1.28$ \\
\hline$k\left(\mathrm{kPa} . \mathrm{s}^{\mathrm{n}}\right)$ & $170.13 \pm 3.06$ & $188.52 \pm 3.55$ & $206.26 \pm 4.97$ \\
\hline$n$ & $0.40 \pm 0.02$ & $0.36 \pm 0.02$ & $0.44 \pm 0.02$ \\
\hline RMS error $(\%)$ & $9.86 \%$ & $7.85 \%$ & $6.68 \%$ \\
\hline
\end{tabular}




\section{FIGURES}

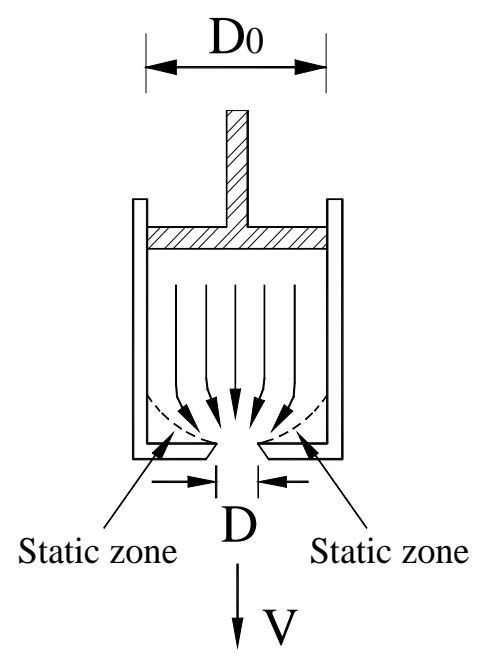

Fig. 1 Schematic illustration of orifice extrusion with paste flow and static zones 


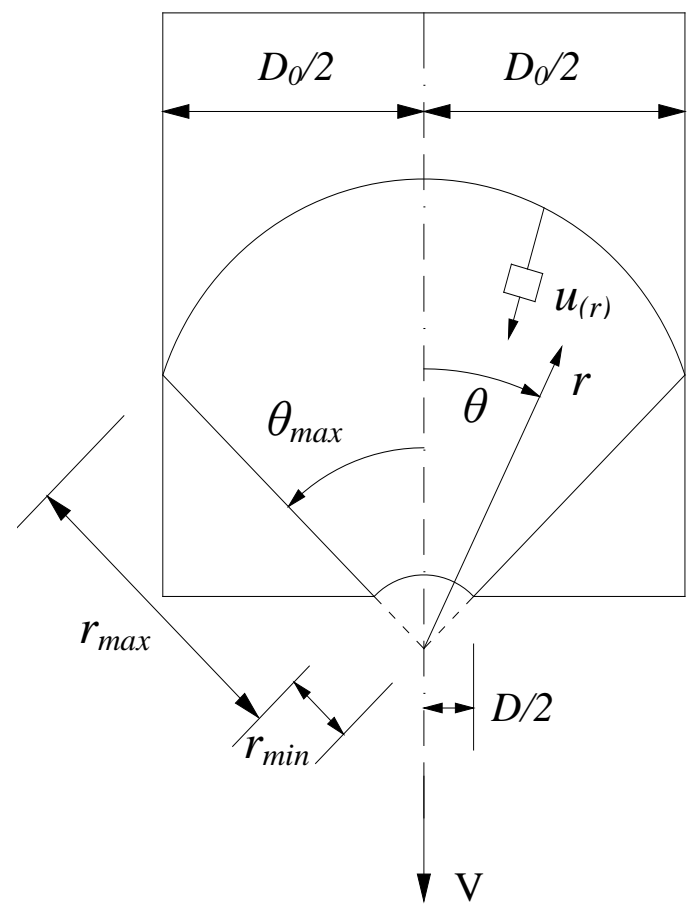

Fig. 2 Schematic diagram of orifice extrusion flow in a spherical coordinate system 


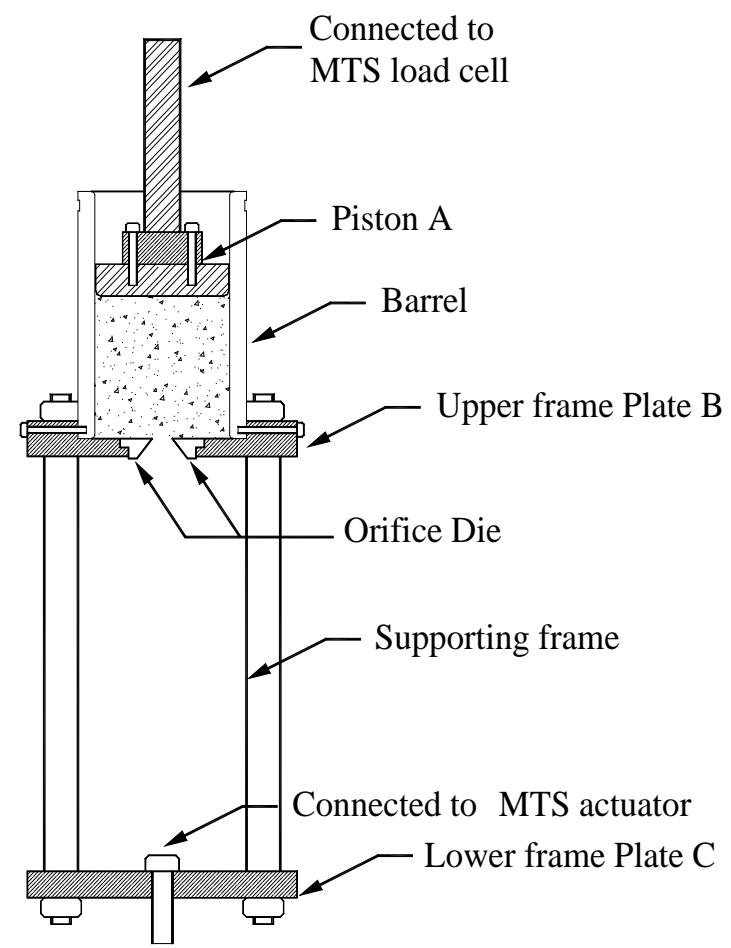

Fig. 3 Schematic diagram of the in-house orifice extruder used for this study 


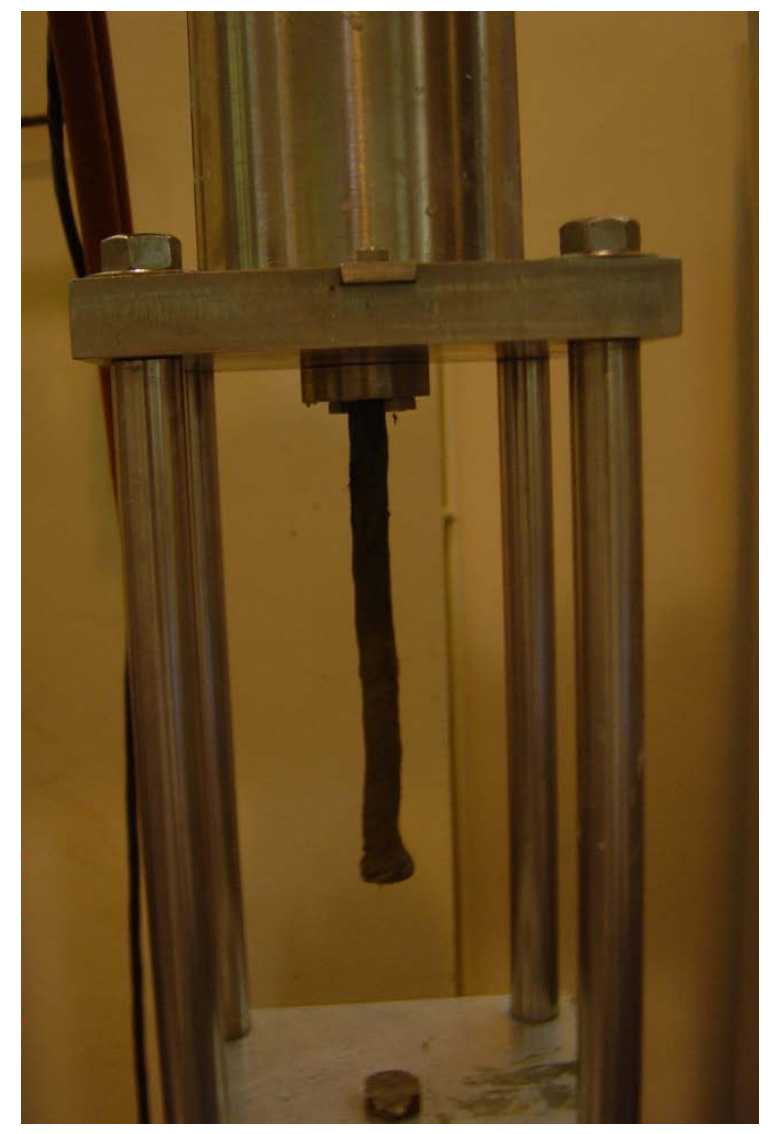

Fig. 4 Orifice extrusion in process 


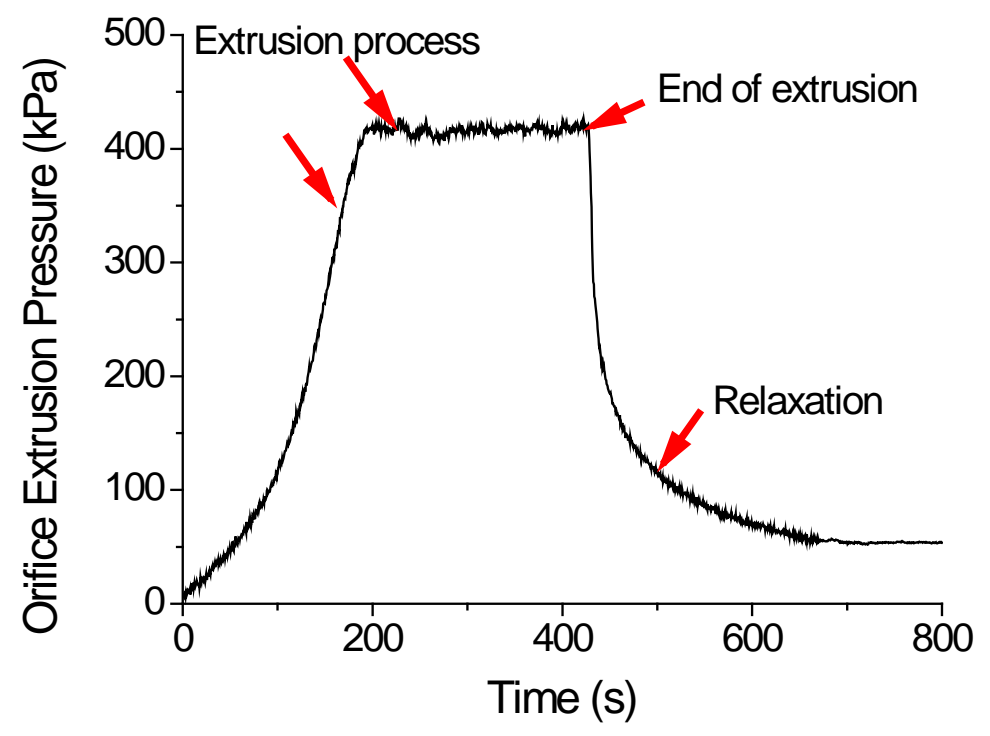

Fig. 5 A typical plot of orifice extrusion and relaxation pressure vs. time 


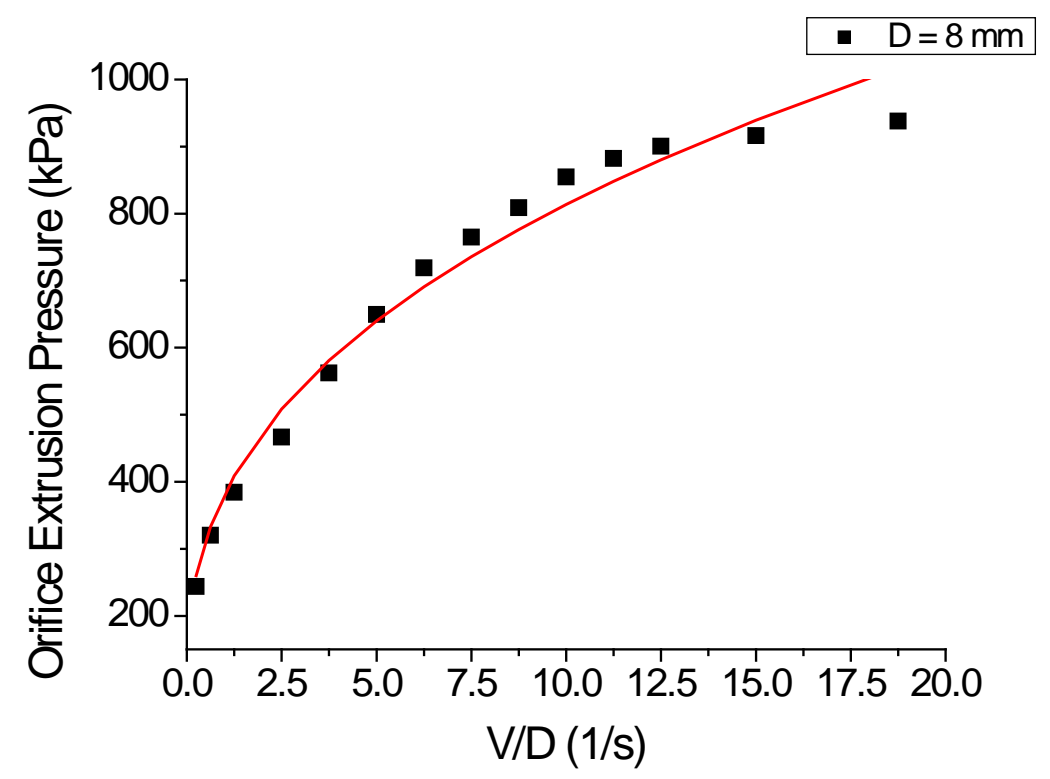

Fig. 6 Orifice extrusion pressure vs. mean shear rate, V/D, and curve fitting to the analytical model (Eq. (15)) for the orifice diameter $\mathrm{D}=8 \mathrm{~mm}$ 


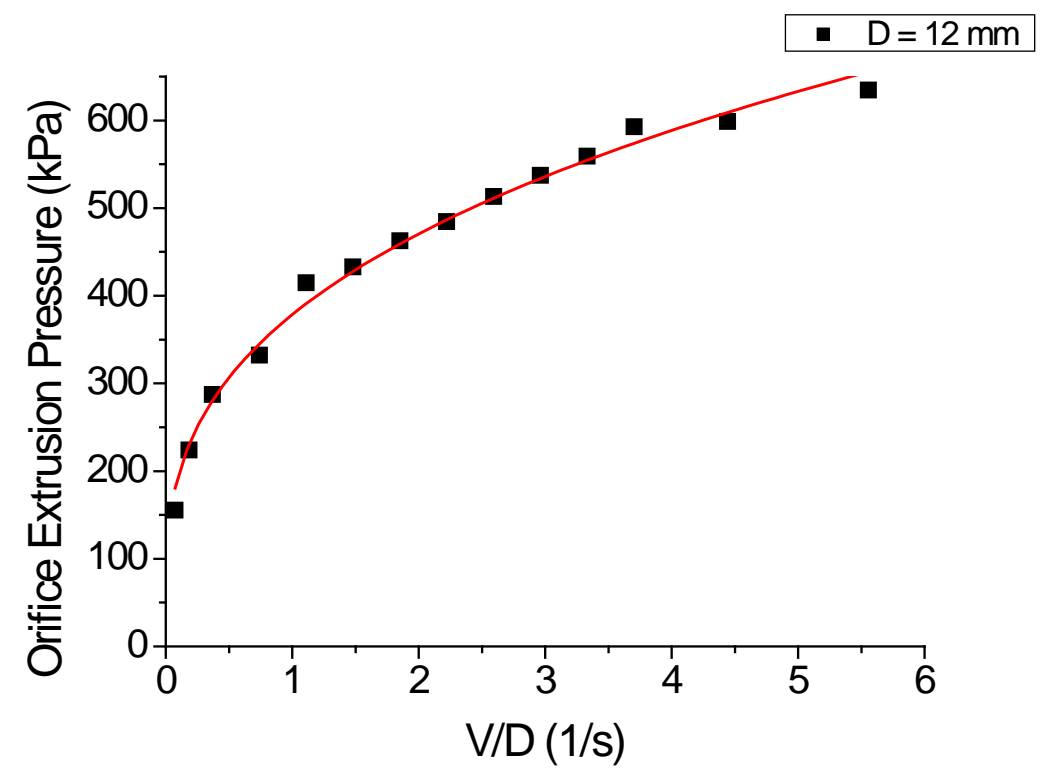

Fig. 7 Orifice extrusion pressure vs. mean shear rate, V/D, and curve fitting to the analytical model (Eq. (15)) for the orifice diameter $\mathrm{D}=12 \mathrm{~mm}$ 


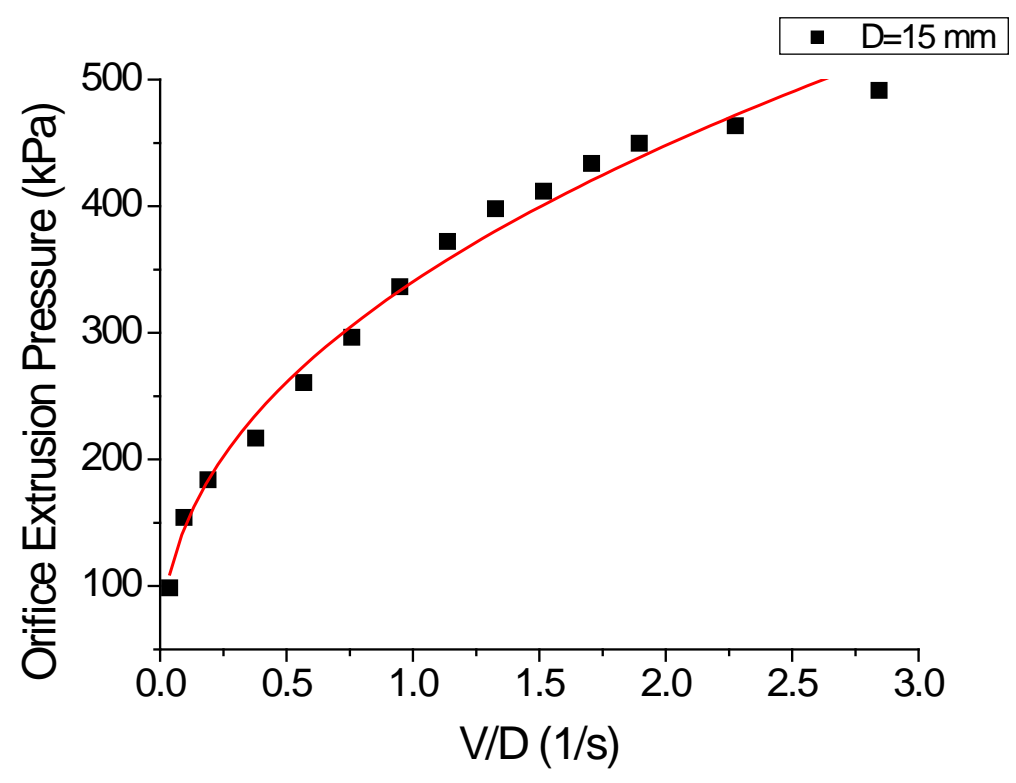

Fig. 8 Orifice extrusion pressure vs. mean shear rate, V/D, and curve fitting to the analytical model (Eq. (15)) for the orifice diameter $\mathrm{D}=15 \mathrm{~mm}$ 